\title{
Map-Based Precision Vehicle Localization in Urban Environments
}

\author{
Jesse Levinson, Michael Montemerlo, Sebastian Thrun \\ Stanford Artificial Intelligence Laboratory \\ \{jessel,mmde,thrun\}@stanford.edu
}

\begin{abstract}
Many urban navigation applications (e.g., autonomous navigation, driver assistance systems) can benefit greatly from localization with centimeter accuracy. Yet such accuracy cannot be achieved reliably with GPS-based inertial guidance systems, specifically in urban settings.

We propose a technique for high-accuracy localization of moving vehicles that utilizes maps of urban environments. Our approach integrates GPS, IMU, wheel odometry, and LIDAR data acquired by an instrumented vehicle, to generate high-resolution environment maps. Offline relaxation techniques similar to recent SLAM methods $[2,10,13,14,21,30]$ are employed to bring the map into alignment at intersections and other regions of self-overlap. By reducing the final map to the flat road surface, imprints of other vehicles are removed. The result is a 2-D surface image of ground reflectivity in the infrared spectrum with $5 \mathrm{~cm}$ pixel resolution.

To localize a moving vehicle relative to these maps, we present a particle filter method for correlating LIDAR measurements with this map. As we show by experimentation, the resulting relative accuracies exceed that of conventional GPS-IMU-odometry-based methods by more than an order of magnitude. Specifically, we show that our algorithm is effective in urban environments, achieving reliable real-time localization with accuracy in the 10centimeter range. Experimental results are provided for localization in GPS-denied environments, during bad weather, and in dense traffic. The proposed approach has been used successfully for steering a car through narrow, dynamic urban roads.
\end{abstract}

\section{INTRODUCTION}

In recent years, there has been enormous interest in making vehicles smarter, both in the quest for autonomously driving cars $[4,8,20,28]$ and for assistance systems that enhance the awareness and safety of human drivers. In the U.S., some 42,000 people die every year in traffic, mostly because of human error [34]. Driver assistance systems such

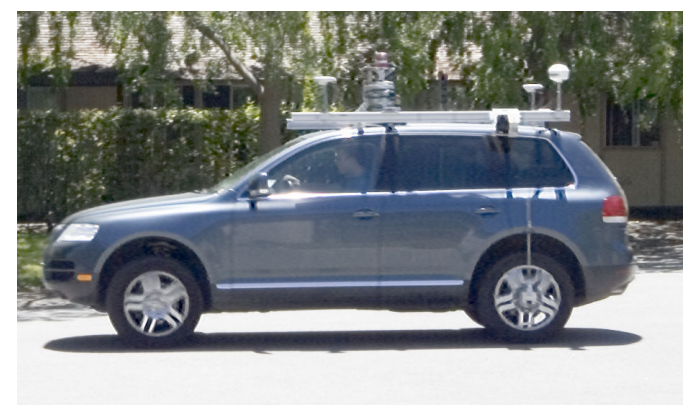

Figure 1. The acquisition vehicle is equipped with a tightly integrated inertial navigation system which uses GPS, IMU, and wheel odometry for localization. It also possesses laser range finders for road mapping and localization. as adaptive cruise control, lane departure warning and lane change assistant all enhance driver safety. Of equal interest in this paper are recent advances in autonomous driving. DARPA recently created the "Urban Challenge" program [5], to meet the congressional goal of having a third of all combat ground vehicles unmanned in 2015. In civilian applications, autonomous vehicles could free drivers of the burden of driving, while significantly enhancing vehicle safety.

All of these applications can greatly benefit from precision localization. For example, in order for an autonomous robot to follow a road, it needs to know where the road is. To stay in a specific lane, it needs to know where the lane is. For an autonomous robot to stay in a lane, the localization requirements are in the order of decimeters. As became painfully clear in the 2004 DARPA Grand Challenge [33], GPS alone is insufficient and does not meet these requirements. Here the leading robot failed after driving off the road because of GPS error.

The core idea of this paper is to augment inertial navigation by learning a detailed map of the environment, and then to use a vehicle's LIDAR sensor to localize relative to this map. In the present paper, maps are 2-D overhead views of the road surface, taken in the infrared spectrum. Such maps capture a multitude of textures in the environment that may be useful for localization, such as lane markings, tire marks, pavement, and vegetating near the road (e.g., grass). The maps are acquired by a vehicle equipped with a state-of-the-art inertial navigation system (with GPS) and multiple SICK laser range finders.

The problem of environment mapping falls into the realm of SLAM (simultaneous localization and mapping), hence there exists a huge body of related work on which our approach builds [3, 11, 12, 24, 22, 26]. SLAM addresses the problem of building consistent environment maps from a moving robotic vehicle, while simultaneously localizing the vehicle relative to these maps. SLAM has been at the core of a number of successful autonomous robot systems [1,29].

At first glance, building urban maps is significantly easier than SLAM, thanks to the availability of GPS most of the time. While GPS is not accurate enough for mapping-as we show in this paper-it nevertheless bounds the localization error, thereby sidestepping hard SLAM problems such as the loop closure problem with unbounded error [17, 18, 31]. However, a key problem rarely addressed in the SLAM literature is that of dynamic environments. Urban environments are dynamic, and for localization to succeed one has to distinguish static aspects of the world (e.g., the road surface) relative to dynamic aspects 


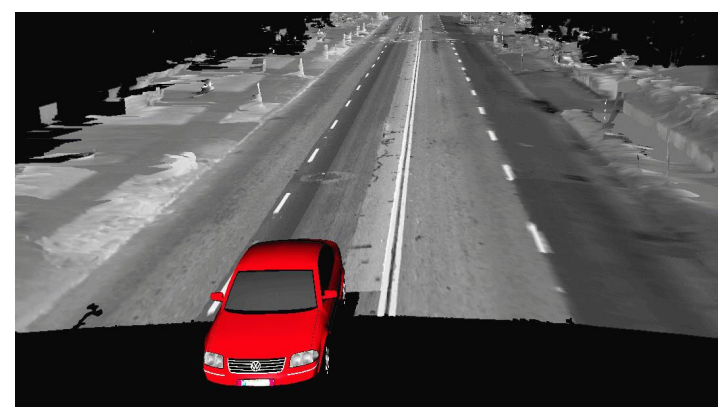

Figure 2. Visualization of the scanning process: the LIDAR scanner acquires range data and infrared ground reflectivity. The resulting maps therefore are 3-D infrared images of the ground reflectivity. Notice that lane markings have much higher reflectivity than pavement.

(e.g., cars driving by). Previous work on SLAM in dynamic environments $[16,19,35]$ has mostly focused on tracking or removing objects that move at the time of mapping, which might not be the case here.

Our approach addresses the problem of environment dynamics, by reducing the map to features that with very high likelihood are static. In particular, using 3-D LIDAR information, our approach only retains the flat road surface; thereby removing the imprints of potentially dynamic objects (e.g., other cars, even if parked). The resulting map is then simply an overhead image of the road surface, where the image brightness corresponds to the infrared reflectivity.

Once a map has been built, our approach uses a particle filter to localize a vehicle in real-time $[6,9,25,27]$. The particle filter analyzes range data in order to extract the ground plane underneath the vehicle. It then correlates via the Pearson product-moment correlation the measured infrared reflectivity with the map. Particles are projected forward through time via the velocity outputs from a tightly-coupled inertial navigation system, which relies on wheel odometry, an IMU and a GPS system for determining vehicle velocity. Empirically, we find that the our system very reliably tracks the location of the vehicle, with relative accuracy of $\sim 10 \mathrm{~cm}$. A dynamic map management algorithm is used to swap parts of maps in and out of memory, scaling precision localization to environments too large to be held in main memory.

Both the mapping and localization processes are robust to dynamic and hilly environments. Provided that the local road surface remains approximately laterally planar in the neighborhood of the vehicle (within $\sim 10 \mathrm{~m}$ ), slopes do not pose a problem for our algorithms.

\section{ROAD MAPPING WITH GRAPHSLAM}

Our mapping method is a version of the GraphSLAM algorithm and related constraint relaxation methods [2, 10, 13, 14, 21, 22, 30].

\section{A. Modeling Motion}

The vehicle transitions through a sequence of poses. In urban mapping, poses are five-dimensional vectors, comprising the $x-y$ coordinates of the robot, along with its heading direction (yaw) and the roll and pitch angle of the vehicle

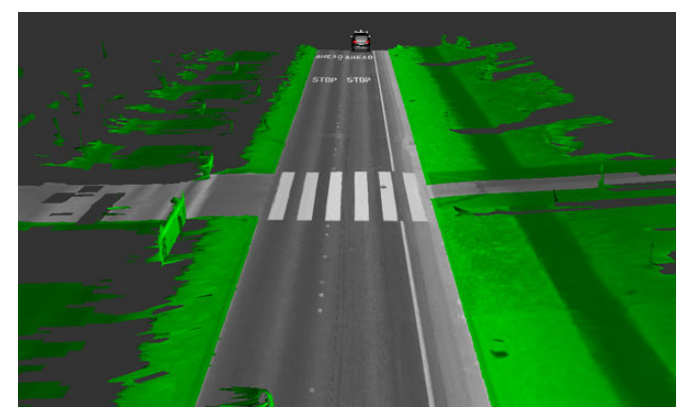

Figure 3. Example of ground plane extraction. Only measurements that coincide with the ground plane are retained; all others are discarded (shown in green here). As a result, moving objects such as car (and even parked cars) are not included in the map. This makes our approach robust in dynamic environments.

(the elevation $z$ is irrelevant for this problem). Let $x_{t}$ denote the pose at time $t$. Poses are linked together through relative odometry data, acquired from the vehicle's inertial guidance system.

$$
x_{t}=g\left(u_{t}, x_{t-1}\right)+\epsilon_{t}
$$

Here $g$ is the non-linear kinematic function which accepts as input a pose $x_{t-1}$ and a motion vector $u_{t}$, and outputs a projected new pose $x_{t}$. The variable $\epsilon_{t}$ is a Gaussian noise variable with zero mean and covariance $R_{t}$. Vehicle dynamics are discussed in detail in [15].

In log-likelihood form, each motion step induces a nonlinear quadratic constraint of the form

$$
\left(x_{t}-g\left(u_{t}, x_{t-1}\right)\right)^{T} R_{t}^{-1}\left(x_{t}-g\left(u_{t}, x_{t-1}\right)\right)
$$

As in $[10,30]$, these constraints can be thought of as edges in a sparse Markov graph.

\section{B. Map Representation}

The map is a 2-D grid which assigns to each $x-y$ location in the environment an infrared reflectivity value. Thus, we can treat the ground map as a orthographic infrared photograph of the ground.

To acquire such a map, multiple laser range finders are mounted on a vehicle, pointing downwards at the road surface (see Fig. 1). In addition to returning the range to a sampling of points on the ground, these lasers also return a measure of infrared reflectivity. By texturing this reflectivity data onto the 3-D range data, the result is a dense infrared reflectivity image of the ground surface, as illustrated in Fig. 2 (this map is similar to vision-based ground maps in [32]).

To eliminate the effect of non-stationary objects in the map on subsequent localization, our approach fits a ground plane to each laser scan, and only retains measurements that coincide with this ground plane. The ability to remove vertical objects is a key advantage of using LIDAR sensors over conventional cameras. As a result, only the flat ground is mapped, and other vehicles are automatically discarded from the data. Fig. 3 illustrates this process. The color labeling in this image illustrates the data selection: all green areas protrude above the ground plane and are discarded. Maps like these can be acquired even at night, as the LIDAR system 
does not rely on external light. This makes he mapping result much less dependent on ambient lighting than is the case for passive cameras.

For any pose $x_{t}$ and any (fixed and known) laser angle relative to the vehicle coordinate frame $\alpha_{i}$, the expected infrared reflectivity can easily be calculated. Let $h_{i}\left(m, x_{t}\right)$ be this function, which calculates the expected laser reflectivity for a given map $m$, a robot pose $x_{t}$, and a laser angle $\alpha_{i}$. We model the observation process as follows

$$
z_{t}^{i}=h_{i}\left(m, x_{t}\right)+\delta_{t}^{i}
$$

Here $\delta_{t}^{i}$ is a Gaussian noise variable with mean zero and noise covariance $Q_{t}$.

In log-likelihood form, this provides a new set of constraints, which are of the form

$$
\left(z_{t}^{i}-h_{i}\left(m, x_{t}\right)\right)^{T} Q_{t}^{-1}\left(z_{t}^{i}-h_{i}\left(m, x_{t}\right)\right)
$$

The unknowns in this function are the poses $\left\{x_{t}\right\}$ and the map $m$.

\section{Latent Variable Extension for GPS}

In outdoor environments, a vehicle can use GPS for localization. GPS offers the convenient advantage that its error is usually limited to a few meters. Let $y_{t}$ denote the GPS signal for time $t$. (For notational convenience, we treat $y_{t}$ as a 3-D vector, with yaw estimate simply set to zero and the corresponding noise covariance in the measurement model set to infinity).

At first glance, one might integrate GPS through an additional constraint in the objective function $J$. The resulting constraints could be of the form

$$
\sum_{t}\left(x_{t}-y_{t}\right)^{T} \Gamma_{t}^{-1}\left(x_{t}-y_{t}\right)
$$

where $\Gamma_{t}$ is the noise covariance of the GPS signal. However, this form assumes that GPS noise is independent. In practice, GPS is subject to systematic noise. because GPS is affected through atmospheric properties, which tend to change slowly with time.

Our approach models the systematic nature of the noise through a Markov chain, which uses GPS bias term $b_{t}$ as a latent variable. The assumption is that the actual GPS measurement is corrupted by an additive bias $b_{t}$, which cannot be observed (hence is latent but can be inferred from data). This model yields constraints of the form

$$
\sum_{t}\left(x_{t}-\left(y_{t}+b_{t}\right)\right)^{T} \Gamma_{t}^{-1}\left(x_{t}-\left(y_{t}+b_{t}\right)\right)
$$

In this model, the latent bias variables $b_{t}$ are subject to a random walk of the form

$$
b_{t}=\gamma b_{t-1}+\beta_{t}
$$

Here $\beta_{t}$ is a Gaussian noise variable with zero mean and covariance $S_{t}$. The constant $\gamma<1$ slowly pulls the bias $b_{t}$ towards zero (e.g., $\gamma=0.999999$ ).

\section{The Extended GraphSLAM Objective Function}

Putting this all together, we obtain the goal function

$$
\begin{aligned}
J= & \sum_{t}\left(x_{t}-g\left(u_{t}, x_{t-1}\right)\right)^{T} R_{t}^{-1}\left(x_{t}-g\left(u_{t}, x_{t-1}\right)\right) \\
& +\sum_{t, i}\left(z_{t}^{i}-h_{i}\left(m, x_{t}\right)\right)^{T} Q_{t}^{-1}\left(z_{t}^{i}-h_{i}\left(m, x_{t}\right)\right) \\
& +\sum_{t}\left(x_{t}-\left(y_{t}+b_{t}\right)\right)^{T} \Gamma_{t}^{-1}\left(x_{t}-\left(y_{t}+b_{t}\right)\right) \\
& +\sum_{t}\left(b_{t}-\gamma b_{t-1}\right)^{T} S_{t}^{-1}\left(b_{t}-\gamma b_{t-1}\right)
\end{aligned}
$$

Unfortunately, this expression cannot be optimized directly, since it involves many millions of variables (poses and map pixels).

\section{E. Integrating Out the Map}

Unfortunately, optimizing $J$ directly is computationally infeasible. A key step in GraphSLAM, which we adopt here, is to first integrate out the map variables. In particular, instead of optimizing $J$ over all variables $\left\{x_{t}\right\},\left\{b_{t}\right\}$, and $m$, we first optimize a modified version of $J$ that contains only poses $\left\{x_{t}\right\}$ and biases $\left\{b_{t}\right\}$, and then compute the most likely map. This is motivated by the fact that, as shown in [30], the map variables can be integrated out in the SLAM joint posterior. Since nearly all unknowns in the system are with the map, this simplification makes the problem of optimizing $J$ much easier and allows it to be solved efficiently.

In cases where a specific surface patch is only seen once during mapping, our approach can simply ignore the corresponding map variables during the pose alignment process, because such patches have no bearing on the pose estimation. Consequently, we can safely remove the associated constraints from the goal function $J$, without altering the goal function for the poses.

Of concern, however, are places that are seen more than once. Those $d o$ create constraint between pose variables from which those places were seen. These constraints correspond to the famous loop closure problem in SLAM [17, 18, 31]. To integrate those map variables out, our approach uses an effective approximation known as map matching [21]. Map matching compares local submaps, in order to find the best alignment.

Our approach implements map matching by first identifying regions of overlap, which will then form the local maps. A region of overlap is the result of driving over the same terrain twice. Formally it is defined as two disjoint sequences of time indices, $t_{1}, t_{2}, \ldots$ and $s_{1}, s_{2}, \ldots$, such that the corresponding grid cells in the map show an overlap that exceeds a given threshold $\theta$.

Once such a region is found, our approach builds two separate maps, one using only data from $t_{1}, t_{2}, \ldots$, and the other only with data from $s_{1}, s_{2}, \ldots$ It then searches for the alignment that maximizes the measurement probability, assuming that both adhere to a single maximum likelihood infrared reflectivity map in the area of overlap.

Specifically, a linear correlation field is computed between these maps, for different $x-y$ offsets between these images. 
Since the poses prior to alignment have already been postprocessed from GPS and IMU data, we find that rotational error between matches is insignificant. Because one or both maps may be incomplete, our approach only computes correlation coefficients from elements whose infrared reflectivity value is known. In cases where the alignment is unique, we find a single peak in this correlation field. The peak of this correlation field is then assumed to be the best estimate for the local alignment. The relative shift is then labeled $\delta_{s t}$, and the resulting constraint of the form above is added to the objective $J$.

This map matching step leads to the introduction of the following constraint in $J$ :

$$
\left(x_{t}+\delta_{s t}-x_{s}\right)^{T} L_{s t}\left(x_{t}+\delta_{s t}-x_{s}\right)
$$

Here $\mathrm{d} \delta_{s t}$ is the local shift between the poses $x_{s}$ and $x_{t}$, and $L_{s t}$ is the strength of this constraint (an inverse covariance). Replacing the map variables in $J$ with this new constraint is clearly approximate; however, it makes the resulting optimization problem tractable.

The new goal function $J^{\prime}$ then replaces the many terms with the measurement model by a small number of between-pose constraints. It is of the form:

$$
\begin{aligned}
J^{\prime}= & \sum_{t}\left(x_{t}-g\left(u_{t}, x_{t-1}\right)\right)^{T} R_{t}^{-1}\left(x_{t}-g\left(u_{t}, x_{t-1}\right)\right) \\
& +\sum_{t}\left(x_{t}-\left(y_{t}+b_{t}\right)\right)^{T} \Gamma_{t}^{-1}\left(x_{t}-\left(y_{t}+b_{t}\right)\right) \\
& +\sum_{t}\left(b_{t}-\gamma b_{t-1}\right)^{T} S_{t}^{-1}\left(b_{t}-\gamma b_{t-1}\right) \\
& +\sum_{t}\left(x_{t}+\delta_{s t}-x_{s}\right)^{T} L_{s t}\left(x_{t}+\delta_{s t}-x_{s}\right)
\end{aligned}
$$

This function contains no map variables, and thus there are orders of magnitude fewer variables in $J^{\prime}$ than in $J . J^{\prime}$ is then easily optimized using CG. For the type of maps shown in this paper, the optimization takes significantly less time on a $\mathrm{PC}$ than is required for acquiring the data. Most of the time is spent in the computation of local map links; the optimization of $J^{\prime}$ only takes a few seconds. The result is an adjusted robot path that resolves inconsistencies at intersections.

\section{F. Computing the Map}

To obtain the map, our approach needs to simply fill in all map values for which one or more measurements are available. In grid cells for which more than one measurement is available, the average infrared reflectivity minimizes the joint data likelihood function.

We notice that this is equivalent to optimize the missing constraints in $J$, denoted $J^{\prime \prime}$ :

$$
J^{\prime \prime}=\sum_{t, i}\left(z_{t}^{i}-h_{i}\left(m, x_{t}\right)\right)^{T} Q_{t}^{-1}\left(z_{t}^{i}-h_{i}\left(m, x_{t}\right)\right)
$$

under the assumption that the poses $\left\{x_{t}\right\}$ are known. Once again, for the type of maps shown in this paper, the computation of the aligned poses requires only a few seconds.

When rendering a map for localization or display, our implementation utilizes hardware accelerated OpenGL to render (a) Map acquired on a sunny day.

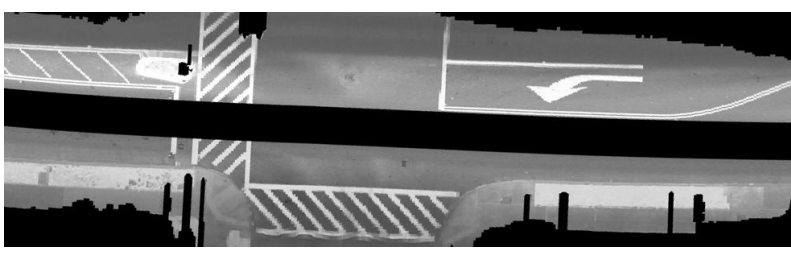

(b) Same road segment on a rainy day.

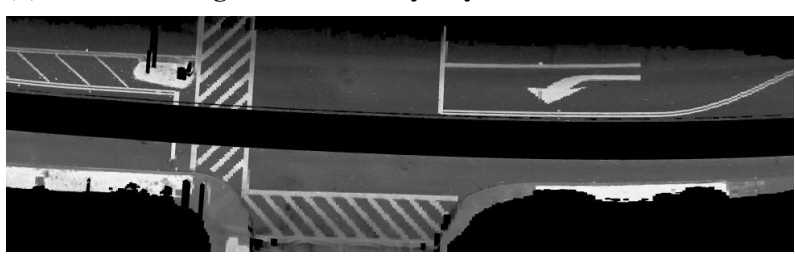

Figure 4. Patch of the map acquired in bright sunlight on a sunny day (top), and at night in heavy rain (middle). By correlating scans with the map, instead of taking absolute differences, the weather-related brightness variation has almost no effect on localization.

smoothly interpolated polygons whose vertices are based on the distances and intensities returned by the three lasers as the robot traverses its trajectory. Even with a low-end graphics card, this process is faster than real-time.

\section{ONLINE LOCALIZATION}

\section{A. Localization with Particle Filters}

Localization uses the map in order to localize the vehicle relative to the map. Localization takes place in real-time, with a $200 \mathrm{~Hz}$ motion update (measurements arrive at $75 \mathrm{~Hz}$ per laser).

Our approach utilizes the same mathematical variable model discussed in the previous section. However, to achieve realtime performance, we utilize a particle filter, known in robotics as Monte Carlo localizer [7]. The particle filter maintains a three-dimensional pose vector ( $x, y$, and yaw); roll and pitch are assumed to be sufficiently accurate as is. The motion prediction in the particle filter is based on inertial velocity measurements, as stated in Sect. II-A.

Measurements are integrated in the usual way, by affecting the importance weight that sets the resampling probability. As in the mapping step, a local ground plane analysis removes measurement that correspond to non-ground objects. Further, measurements are only incorporated for which the corresponding map is defined, that is, for which a prior reflectivity value is available in the map. For each measurement update, the importance weight of the $k$-th particle is given by

$$
\begin{aligned}
w_{t}^{[k]}= & \exp \left\{-\frac{1}{2}\left(x_{t}^{[k]}-y_{t}\right)^{T} \Gamma_{t}^{-1}\left(x_{t}^{[k]}-y_{t}\right)\right\} \\
& \left(\operatorname{corr}\left[\left(\begin{array}{c}
h_{1}\left(m, x_{t}^{[k]}\right) \\
\vdots \\
h_{180}\left(m, x_{t}^{[k]}\right)
\end{array}\right),\left(\begin{array}{c}
z_{t}^{1} \\
\vdots \\
z_{t}^{180}
\end{array}\right)\right]+1\right)
\end{aligned}
$$

Here, corr() is the Pearson product-moment correlation. The actual resampling takes place at a much lower frequency than the measurement/pose updates, to reduce variance. As usual, importance weight updates are integrated multiplicatively. To avoid catastrophic localization errors, a small number of particles are continuously drawn from current GPS pose estimate. 
This "sensor resetting" trick was proposed in [23]. GPS, when available, is also used in the calculation of the measurement likelihood to reduce the danger of particles moving too far away from the GPS location.

One complicating factor in vehicle localization is weather. As illustrated in Fig. 4, the appearance of the road surface is affected by rain, in that wet surfaces tend to reflect less infrared laser light than do dry ones. To adjust for this effect, the particle filter normalizes the brightness and standard deviation for each individual range scan, and also for the corresponding local map stripes. This normalization transforms the least squares difference method into the computation of the Pearson product-moment correlation with missing variables (empty grid cells in the map). Empirically, we find that this local normalization step is essential for robust performance .

We also note that the particle filter can be run entirely without GPS, where the only reference to the environment is the map. Some of our experiments are carried out in the absence of GPS, to illustrate the robustness of our approach in situations where conventional GPS-based localization is plainly inapplicable.

\section{B. Data Management}

Maps of large environments at 5-cm resolution occupy a significant amount of memory. We have implemented two methods to reduce the size of the maps and to allow relevant data to fit into main memory.

When acquiring data in a moving vehicle, the rectangular area which circumscribes the resulting laser scans grows quadratically with distance, despite that the data itself grows only linearly. In order to avoid a quadratic space requirement, we break the rectangular area into a square grid, and only save squares for which there is data. When losslessly compressed, the grid images require approximately $10 \mathrm{MB}$ per mile of road at $5-\mathrm{cm}$ resolution. This would allow a 200GB hard drive to hold 20,000 miles of data.

Although the data for a large urban environment can fit on hard drive, it may not all be able to fit into main memory. Our particle filter maintains a cache of image squares near the vehicle, and thus requires a constant amount of memory regardless of the size of the overall map.

\section{EXPERIMENTAL RESULTS}

We conducted extensive experiments with the vehicle shown in Fig. 1. This vehicle is equipped with a state-of-the-art inertial navigation system (GPS, inertial sensors, and wheel odometry), and three down-facing laser range finders: one facing the left side, one facing the right side, and one facing the rear. In all experiments, we use a pixel resolution of $5 \mathrm{~cm}$.

\section{A. Mapping}

We tested the mapping algorithm successfully on a variety of urban roads. One of our testing environments is an urban area, shown in Fig. 5. This image shows an aerial view of the testing environment, with the map overlayed in red. To generate this map, our algorithm automatically identified and aligned several hundreds match points in a total of 32 loops. It corrected the trajectory and output consistent imagery at $5-\mathrm{cm}$

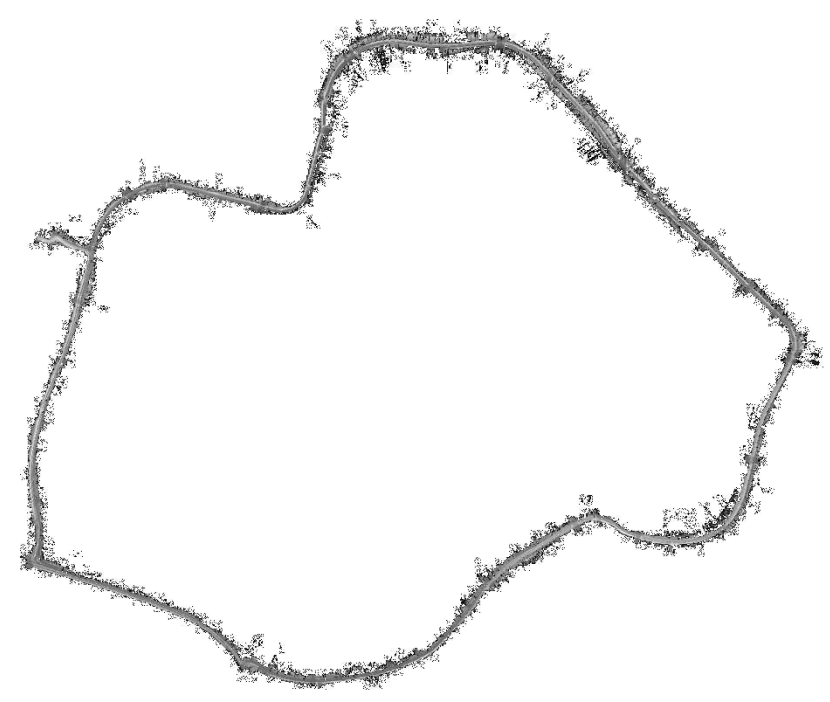

Figure 6. Campus Dr., a loop around Stanford 4 miles in circumference. To acquire this map, we drove the same loop twice, filling in gaps that are occluded by moving vehicles.

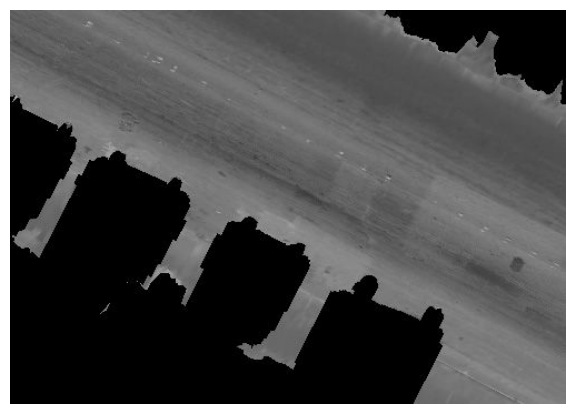

Figure 7. Map with many parked cars. Notice the absence of lane markers.

resolution. Fig. 7 shows a close-up of this map in a residential area lined with parked cars, whose positions changed between different data runs.

One of the key results of the map alignment process is the removal of the so-called "ghosting" effects. Ghosting occurs in the absence of the map alignment step, where the location of measurements are set by GPS alone. Fig. 8a shows an example of ghosting, in which features occur twice in the map. Clearly, such a map is too inaccurate for precision localization. Fig. $8 \mathrm{~b}$ shows the adjusted map, generated with the approach described in this paper. Here the ghosting effect has disappeared, and the map is locally consistent.

Figure 6 shows a large loop which is 4 miles in circumference. Our algorithm automatically identified and aligned 148 match points during the second traversal of the loop. In this experiment, the average magnitude of the corrections calculated by the matching algorithm for these two loops is $35 \mathrm{~cm}$, indicating that without corrections, the two loops are quite poorly aligned, even with post-processed poses.

An interesting result is that areas missing in the first traversal can be filled in during the second. Fig. 9 shows three local maps. The map from the first traversal has a hole caused by oncoming traffic. The center map in this figure is the GPS 


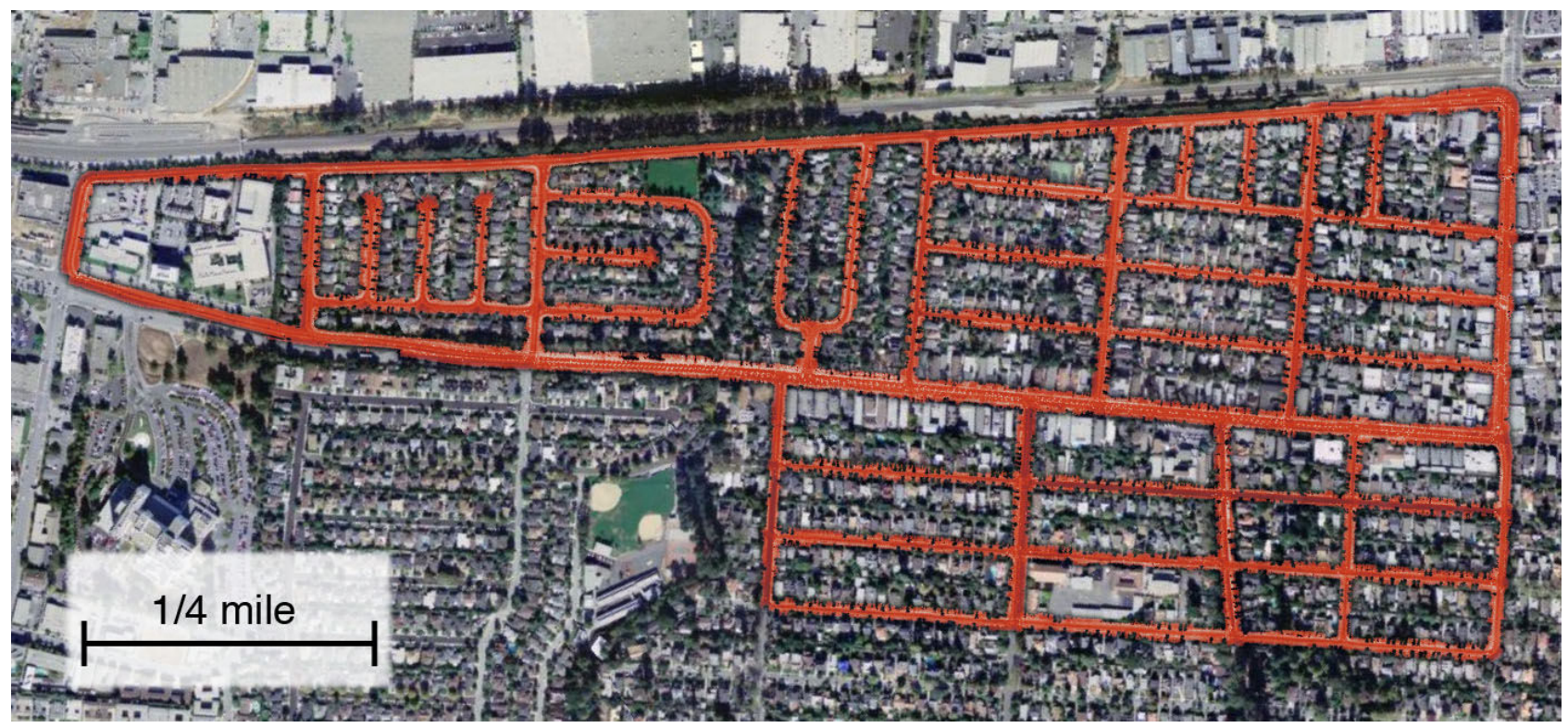

Figure 5. (Best viewed in color) Aerial view of Burlingame, CA. Regions of overlap have been adjusted according to the methods described in this paper. Maps of this size tend not to fit into main memory, but are swapped in from disk automatically during driving.

(a) GPS leads to ghosting

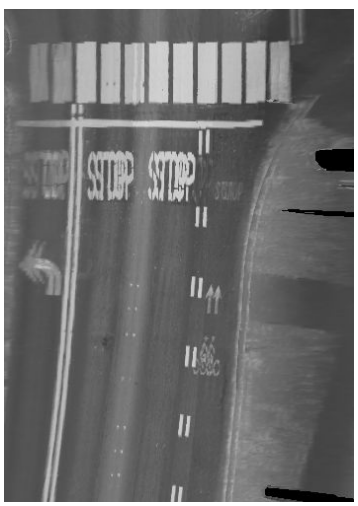

(b) Our method: No ghosting

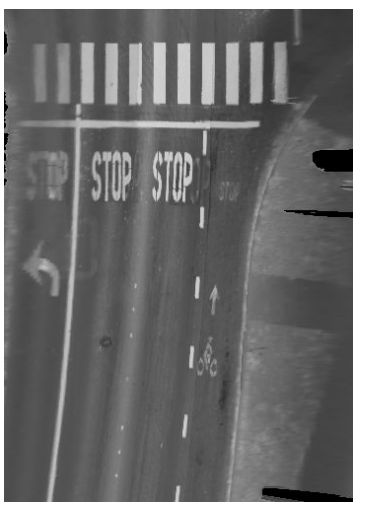

Figure 8. Infrared reflectivity ground map before and after SLAM optimization. Residual GPS drift can be seen in the ghost images of the road markings (left). After optimization, all ghost images have been removed (right).

aligned map with the familiar ghosting effect. On the right is the fused map with our alignment procedure, which possesses no hole and exhibits no ghosting.

The examples shown here are representative of all the maps we have acquired so far. We find that even in dense urban traffic, the alignment process is robust to other cars and non-stationary obstacles. No tests were conducted in open featureless terrain (e.g., airfields without markings or texture), as we believe those are of low relevance for the stated goal of urban localization.

\section{B. Localization}

Our particle filter is adaptable to a variety of conditions. In the ideal case, the vehicle contains an integrated GPS/IMU system, which provides locally consistent velocity estimates and global pose accuracy to within roughly a meter. In our (a) Map with hole

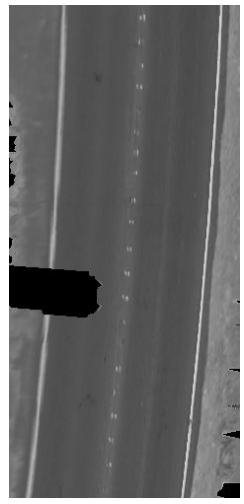

(b) Ghosting

(c) Our approach
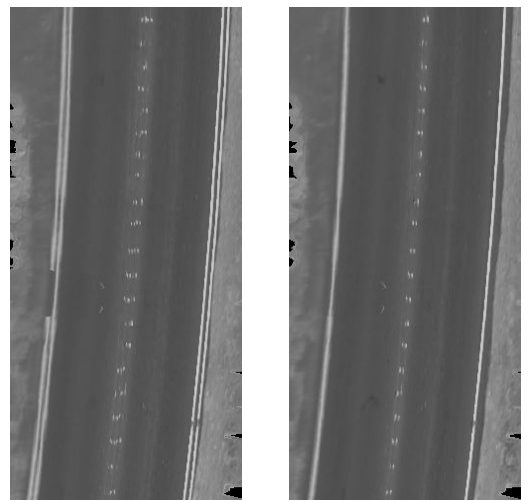

Figure 9. Filtering dynamic objects from the map leaves holes (left). These holes are often filled if a second pass is made over the road, but ghost images remain (center). After SLAM, the hole is filled and the ghost image is removed.

tests in a variety of urban roads, our GPS-equipped vehicle was able to localize in real-time relative to our previously created maps with errors of less than $10 \mathrm{~cm}$, far exceeding the accuracy with GPS alone.

We ran a series of experiments to test localization relative to the learned map. All localization experiments used separate data from the mapping data; hence the environment was subject to change that, at times, was substantial. In fact, the map in Fig. 5 was acquired at night, but all localization experiments took place during daylight. In our experimentation, we used between 200 and 300 particles.

Fig. 10 shows an example path that corresponds to 20 minutes of driving and localization. During this run, the average disagreement between real-time GPS pose and our method was $66 \mathrm{~cm}$. Manual alignment of the map and the 


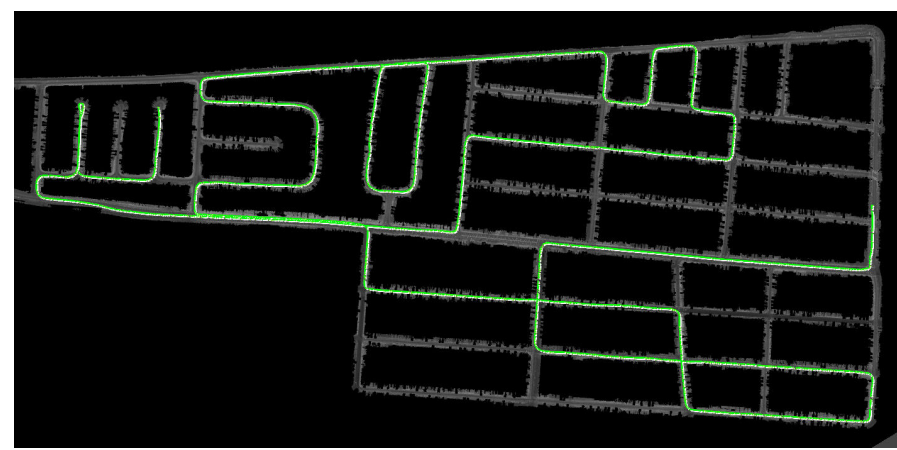

Figure 10. (Best viewed in color) Typical driving path during localization shown in green, and overlayed on a previously built map, acquired during 20 minutes of driving. For this and other paths, we find that the particle filter reliably localizes the vehicle.

(a) GPS localization induces $\geq 1$ meter of error.

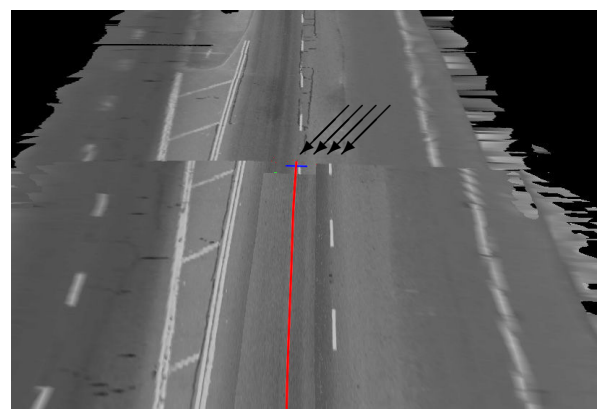

(b) No noticeable error in particle filter localization.

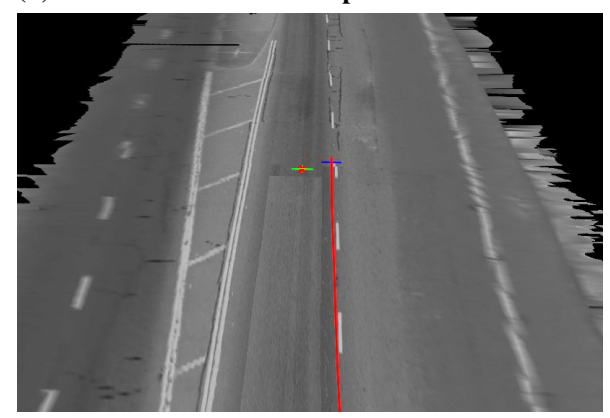

Figure 11. (a) GPS localization is prone to error, even (as shown here) with a high-end integrated inertial system and differential GPS using a nearby stationary antenna. (b) The particle filter result shows no noticeable error.

incoming LIDAR measurements suggests that the lateral error was almost always within $10 \mathrm{~cm}$. Occasionally, errors were larger; when turning, errors were sometimes as large as $30 \mathrm{~cm}$. However, this is still well below the error rate of the GPSbased inertial system.

A typical situation is shown in Fig. 11. Fig. 11a superimposes data acquired online by the vehicle and the previously acquired map. The error here is greater than one meter. Fig. 11b shows the result with our approach, where the error is below the map resolution. The red line in both figures corresponds to the path of the localizing vehicle.

One of the key aspects of the localization method is its ability to localize entirely in the absence of GPS. For the following experiments, we switched off the GPS receiver,

\begin{tabular}{|r|r|r|}
\hline \multicolumn{3}{|c|}{ Stanford Ave. } \\
\hline \hline $\begin{array}{r}\text { Distance } \\
\text { Traveled }(\mathrm{m})\end{array}$ & $\begin{array}{r}\text { Our Error } \\
(\mathrm{cm})\end{array}$ & $\begin{array}{r}\text { Odometry } \\
\text { Error }(\mathrm{cm})\end{array}$ \\
\hline 50 & 7 & 98 \\
100 & 3 & 149 \\
150 & 35 & 0 \\
200 & 13 & 8 \\
250 & 4 & 133 \\
300 & 22 & 272 \\
350 & 8 & 428 \\
400 & 23 & 589 \\
450 & 13 & 783 \\
499 & 10 & 924 \\
\hline
\end{tabular}

Figure 12. This table compares the accuracy of pose estimation in the absence of GPS or IMU data. The right column is obtained by odometry only; the center by particle filter localization relative to the map. Clearly, odometry alone accumulates error. Our approach localizes reliably without any GPS or IMU.

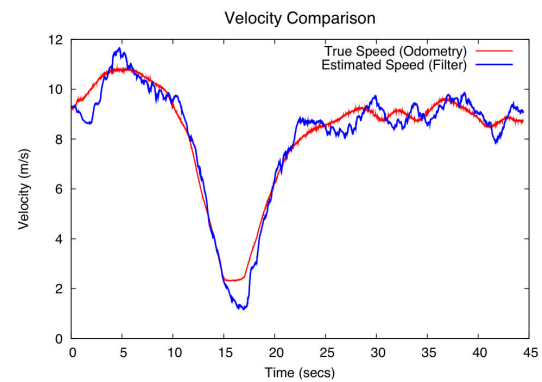

Figure 13. Estimated velocity from odometry and our particle filter, not using odometry or GPS. The strong correspondence illustrates that a particle filter fed with laser data alone can determine the vehicle velocity, assuming a previously acquired map. Note that in this case, accurate velocity estimation subsumes accurate position estimation.

and exclusively used wheel odometry and steering angle for localization. Clearly, odometry alone eventually diverges from the true position. Nevertheless, our localization method reliable tracks the location of the car.

This is illustrated by the result in Fig. 12, which quantitatively compares the accuracy of the particle filter localizer with odometry data. In both cases, the filters are initialized with the correct pose. Error is measured along 10 hand-labeled reference points in 50 meter increments.

Not surprisingly, the odometry estimates grows quickly as the vehicle moves, whereas our method results in a small error. The error is slightly larger than in the experiments above, due to the absence of GPS in the forward projection step of the particles. Nevertheless, this experiment illustrates successful tracking in the absence of GPS.

Finally, an extreme test of our software's ability to localize using laser data was performed by disallowing any motion or position data whatsoever in the motion update. Thus odometry, IMU, and GPS measurements were all ignored. In this case, the particles' state vector included $\mathrm{x}$ and $\mathrm{y}$ position, yaw, steering angle, velocity, and acceleration. The particles were initialized near the true position, and reasonable values were assumed for the rate of change of the control parameters. Remarkably, our system was able to track the position and the velocity of the vehicle using nothing but laser data (see Fig. 13). 


\section{Autonomous Driving}

In a series of experiments, we used our approach for semiautonomous urban driving. In most of these experiments, gas and brakes were manually operated, but steering was controlled by a computer. The vehicle followed a fixed reference trajectory on a university campus. In some cases, the lane width exceeded the vehicle width by less than 2 meters.

Using the localization technique described here, the vehicle was able to follow this trajectory on ten out of ten attempts without error; never did our method fail to provide sufficient localization accuracy. Similar experiments using only GPS consistently failed within meters, illustrating that GPS alone is insufficient for autonomous urban driving. We view the ability to use our localization method to steer a vehicle in an urban environment as a success of our approach.

\section{Conclusions}

Localization is a key enabling factor for urban robotic operation. With accurate localization, autonomous cars can perform accurate lane keeping and obey traffic laws (e.g., stop at stop signs). Although nearly all outdoor localization work is based on GPS, GPS alone is insufficient to provide the accuracy necessary for urban autonomous operation.

This paper presented a localization method that uses an environment map. The map is acquired by an instrumented vehicle that uses infrared LIDAR to measure the 3-D structure and infrared reflectivity of the environment. A SLAM-style relaxation algorithm transforms this data into a globally consistent environment model with non-ground objects removed. A particle filter localizer was discussed that enables a moving vehicle to localize in real-time, at $200 \mathrm{~Hz}$.

Extensive experiments in urban environments suggest that the localization method surpasses GPS in two critical dimensions: accuracy and availability. The proposed method is significantly more accurate than GPS; its relative lateral localization error to the previously recorded map is mostly within $5 \mathrm{~cm}$, whereas GPS errors are often in the order of 1 meter. Second, our method succeeds in GPS-denied environments, where GPS-based systems fail. This is of importance to navigation in urban canyons, tunnels, and other structures that block or deflect GPS signals.

The biggest disadvantage of our approach is its reliance on maps. While road surfaces are relatively constant over time, they still may change. In extreme cases, our localization technique may fail. While we acknowledge this limitation, the present work does not address it directly. Possible extensions would involve filters that monitor the sensor likelihood, to detect sequences of "surprising" measurements that may be indicative of a changed road surface. Alternatively, it may be possible to compare GPS and map-based localization estimates to spot positioning errors. Finally, it may be desirable to extend our approach to incorporate 3-D environment models beyond the road surface for improved reliability and accuracy, especially on unusually featureless roads.

\section{REFERENCES}

[1] C. Baker, A. Morris, D. Ferguson, S. Thayer, C. Whittaker, Z. Omohundro, C. Reverte, W. Whittaker, D. Hähnel, and S. Thrun. A campaign in autonomous mine mapping. In ICRA 2004.
[2] M. Bosse, P. Newman, J. Leonard, M. Soika, W. Feiten, and S. Teller Simultaneous localization and map building in large-scale cyclic environments using the atlas framework. IJRR, 23(12), 2004.

[3] P. Cheeseman and P. Smith. On the representation and estimation of spatial uncertainty. IJR, 5, 1986.

[4] DARPA. DARPA Grand Challenge rulebook, 2004. On the Web at http://www.darpa.mil/grandchallenge05/Rules_8oct04.pdf.

[5] DARPA. DARPA Urban Challenge rulebook, 2006. On the Web at http://www.darpa.mil/grandchallenge/docs/Urban_Challenge_Rules_ 121106.pdf.

[6] F. Dellaert, W. Burgard, D. Fox, and S. Thrun. Using the condensation algorithm for robust, vision-based mobile robot localization. ICVP 1999.

[7] F. Dellaert, D. Fox, W. Burgard, and S. Thrun. Monte Carlo localization for mobile robots. ICRA 1999.

[8] E.D. Dickmanns. Vision for ground vehicles: history and prospects IJVAS, 1(1) 2002

[9] A Doucet. On sequential simulation-based methods for Bayesian filtering. TR CUED/F-INFENG/TR 310, Cambridge University, 1998.

[10] T. Duckett, S. Marsland, and J. Shapiro. Learning globally consistent maps by relaxation. ICRA 2000 .

[11] H.F. Durrant-Whyte. Uncertain geometry in robotics. IEEE TRA, 4(1), 1988.

[12] A. Eliazar and R. Parr. DP-SLAM: Fast, robust simultaneous localization and mapping without predetermined landmarks. IJCAI 2003.

[13] J. Folkesson and H. I. Christensen. Robust SLAM. ISAV 2004.

[14] U. Frese, P. Larsson, and T. Duckett. A multigrid algorithm for simultaneous localization and mapping. IEEE Transactions on Robotics, 2005.

[15] T.D. Gillespie. Fundamentals of Vehicle Dynamics. SAE Publications, 1992.

[16] J. Guivant, E. Nebot, and S. Baiker. Autonomous navigation and map building using laser range sensors in outdoor applications. JRS, 17(10), 2000.

[17] J.-S. Gutmann and K. Konolige. Incremental mapping of large cyclic environments. CIRA 2000

[18] D. Hähnel, D. Fox, W. Burgard, and S. Thrun. A highly efficient FastSLAM algorithm for generating cyclic maps of large-scale environments from raw laser range measurements. IROS 2003.

[19] D. Hähnel, D. Schulz, and W. Burgard. Map building with mobile robots in populated environments. IROS 2002.

[20] M. Hebert, C. Thorpe, and A. Stentz. Intelligent Unmanned Ground Vehicles. Kluwer, 1997.

[21] K. Konolige. Large-scale map-making. AAAI, 2004.

[22] B. Kuipers and Y.-T. Byun. A robot exploration and mapping strategy based on a semantic hierarchy of spatial representations. JRAS, 8, 1991

[23] S. Lenser and M. Veloso. Sensor resetting localization for poorly modelled mobile robots. ICRA 2000.

[24] J. Leonard, J.D. Tardós, S. Thrun, and H. Choset, editors. ICRA Workshop W4, 2002.

[25] J. Liu and R. Chen. Sequential monte carlo methods for dynamic systems. Journal of the American Statistical Association, 93. 1998.

[26] M.A. Paskin. Thin junction tree filters for simultaneous localization and mapping. IJCAI 2003.

[27] M. Pitt and N. Shephard. Filtering via simulation: auxiliary particle filter. Journal of the American Statistical Association, 94, 1999.

[28] D. Pomerleau. Neural Network Perception for Mobile Robot Guidance. Kluwer, 1993

[29] C. Thorpe and H. Durrant-Whyte. Field robots. ISRR 2001

[30] S. Thrun and M. Montemerlo. The GraphSLAM algorithm with applications to large-scale mapping of urban structures. IJRR, 25(5/6), 2005.

[31] N. Tomatis, I. Nourbakhsh, and R. Siegwart. Hybrid simultaneous localization and map building: closing the loop with multi-hypothesis tracking. ICRA 2002

[32] I. Ulrich and I. Nourbakhsh. Appearance-based obstacle detection with monocular color vision. AAAI 2000.

[33] C. Urmson, J. Anhalt, M. Clark, T. Galatali, J.P. Gonzalez, J. Gowdy, A. Gutierrez, S. Harbaugh, M. Johnson-Roberson, H. Kato, P.L. Koon, K. Peterson, B.K. Smith, S. Spiker, E. Tryzelaar, and W.L. Whittaker. High speed navigation of unrehearsed terrain: Red Team technology for the Grand Challenge 2004. TR CMU-RI-TR-04-37, 2004.

[34] Bureau of Transportation Statistics U.S. Department of Transportation. Annual report, 2005.

[35] C.-C. Wang, C. Thorpe, and S. Thrun. Online simultaneous localization and mapping with detection and tracking of moving objects: Theory and results from a ground vehicle in crowded urban areas. ICRA 2003. 